\title{
General transformation for the reduced invisibility cloak
}

\author{
Huanyang Chen, ${ }^{*}$ Jack Ng, C. W. Jeffrey Lee, Yun Lai, and C. T. Chan ${ }^{\dagger}$ \\ Department of Physics, The Hong Kong University of Science and Technology, Clear Water Bay, Kowloon, Hong Kong, China
}

(Received 15 May 2009; published 19 August 2009)

\begin{abstract}
We present a generalized perspective for "reduced" invisibility cloaks, which formulates a general way to "reduce" the cloak so that it can be realized more easily. We show explicitly that several published reduced cloak designs are in fact special cases of a more general transformation. Furthermore, we showed that a cylindrical reduced cloak is linked to a cylindrical isotropic inhomogeneous object by exactly the coordinate transformation that one applies on an ideal cloak. This perspective also suggests an alternative but easier way to calculate the scattering properties of the reduced cloaks.
\end{abstract}

DOI: 10.1103/PhysRevB.80.085112

PACS number(s): 41.20.Jb, 42.25.Fx, 42.79.-e, 42.25.Gy

Transformation optics ${ }^{1,2}$ is a powerful and versatile theoretical tool that enables the design of various kinds of novel metamaterial devices. The most celebrated example is the invisibility cloaks. ${ }^{3-18}$ Other examples include field concentrators, ${ }^{19}$ field rotators, ${ }^{20-22}$ electromagnetic wormholes, ${ }^{23}$ impedance-matched hyperlenses, ${ }^{24}$ field shifters, ${ }^{25,26}$ anticloaks, ${ }^{27}$ cylindrical superlenses, ${ }^{28}$ superscatterers, ${ }^{29}$ superabsorbers, ${ }^{30}$ and remote cloaks (or invisibility umbrellas). ${ }^{31,32}$ However, the experimental fabrication of these novel devices requires sophisticated man-made metamaterial, which may not be always feasible to make. For this reason, researchers have attempted to "reduce" the complexity of the required constitutive parameters. The functionalities of these reduced devices, such as the reduced cloak, ${ }^{3,5-7}$ are slightly less perfect compared to the original ideal devices, but they are designed to perform satisfactorily while having the advantage that they are much easier to fabricate. Indeed, the "reduced devices" will become those that shall be useful for real applications as the ideal devices are probably too difficult or expensive to make. So, there is a good reason that much effort ${ }^{8,9,11-13}$ has been devoted to study the reduced cloaks. For example, a reduced cloak working in the microwave frequency was realized experimentally in 2006. ${ }^{5}$ More recently, the "ground-plane cloak" 16

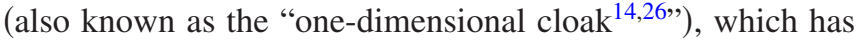
a broad working frequency spectrum, was also realized.

For simplicity, we shall only consider the two dimensional case in this paper. The spatially varying constitutive parameters for the transverse electric (TE) ideal cloak are given by

$$
\mu_{r}=\frac{f(r)}{r f^{\prime}(r)}, \mu_{\theta}=\frac{r f^{\prime}(r)}{f(r)}, \varepsilon_{z}=\frac{f(r) f^{\prime}(r)}{r},
$$

where $f(r)$ is the mapping function. The constitutive parameters for transverse magnetic (TM) ideal cloak can be obtained by making the substitution $\left\{\mu_{r} \rightarrow \varepsilon_{r}, \mu_{\theta} \rightarrow \varepsilon_{\theta}, \varepsilon_{z}\right.$ $\left.\rightarrow \mu_{z}\right\}$ in (1). By varying the functional form, we can achieve a varieties of functionalities such as the concentrator ${ }^{21}$ (or the impedance-matched hyperlens ${ }^{24}$ ), the anticloak ${ }^{27}$ and the superscatterer ${ }^{29}$ cylindrical superlenses ${ }^{28}$ etc. However, these parameters involve spatially varying, anisotropy permittivity, and permeability, which can be formidably difficult to fabricate. Consequently, it is essential for one to "reduce" or simplify these parameters for real experimental implementation. One important criteria of "reducing" the cloak, as described by Eq. (1), ${ }^{3,6,7}$ is that while one simplifies the constitutive parameters, one must preserve the principal refractive indices

$$
n_{r}^{2}=\mu_{\theta} \varepsilon_{z}=\left[f^{\prime}(r)\right]^{2}, n_{\theta}^{2}=\mu_{r} \varepsilon_{z}=[f(r) / r]^{2} .
$$

Clearly this could be achieved by the following set of constitutive parameters:

$$
\mu_{r}=g(r) \frac{f(r)}{r f^{\prime}(r)}, \mu_{\theta}=g(r) \frac{r f^{\prime}(r)}{f(r)}, \varepsilon_{z}=\frac{1}{g(r)} \frac{f(r) f^{\prime}(r)}{r},
$$

where we have introduced an arbitrary function $g(r)$ into (1). For $g(r)=1$, the parameters in Eq. (3) are precisely that of the ideal cloak. We can "reduce" the cloak by choosing a particular $g(r)$ in Eq. (3) that simplifies the constitutive parameters. Moreover, since Eq. (3) generally fulfills the reducing criteria of Eq. (2), any reduced cloak can be considered as a special case of Eq. (3).

We shall next show that while the original ideal cloak is derived from mapping a virtual cylindrical space of $\varepsilon=\mu$ $=1$ to a cylindrical annular region of constitutive parameters (1), a reduced cloak is derived from mapping a virtual cylindrical space of isotropic inhomogeneous constitutive parameters to a cylindrical annular region of constitutive parameters (3). Suppose in the virtual space $(\rho, \theta)$, there is an isotropic inhomogeneous cylindrical object in vacuum or air with permittivity

$$
\varepsilon=1 / g\left[f^{-1}(\rho)\right]
$$

and permeability

$$
\mu=g\left[f^{-1}(\rho)\right]
$$

By applying exactly the same coordinate transformation [i.e., making use of the mapping function $f(r)]$ to (4) and (5) as in the case of the ideal cloak, it can be shown that the constitutive parameters for the transformed media in the real space are exactly that of (3). ${ }^{2,33}$ Consequently, as far as the far field (i.e., $r>b$ ) scattering pattern is concerned, any cylindrical reduced cloak can be considered as equivalent to an isotropic inhomogeneous cylindrical object. In the special case of an ideal cloak, the cylindrical object becomes homogeneous. This perspective can have several implications. First, our ap- 
proach can delineate why different kinds of the reduced cloaks have different properties or functionalities, as we can treat the reduced cloak as an equivalent isotropic inhomogeneous object. So this perspective provides an alternative way to understand and calculate the functionalities of the reduced cloaks. Second, due to the fact that the constitutive parameters for the reduced cloak are anisotropic, the calculation, and understanding of the scattering properties of the cloak can be difficult. ${ }^{11-13}$ In this case, the finite element method simulation is usually employed, ${ }^{11,12}$ but it is more satisfying to use analytical theories as far as we can and numerical simulations are limited to small scale systems only. In this paper, we show that the scattering properties of the reduced cloak are equivalent to an isotropic inhomogeneous object, and thus the scattering properties may be computed with ease, and at a considerably higher accuracy and efficiency. For example, one can simply treat the object as a stratified cylindrical object with each layer being made of very thin isotropic and homogenous material. In this case, standard methods, like the transfer matrix method (TMM), ${ }^{34}$ can be applied. We note that although the TMM also works for an anisotropic reduced cloak, but the labor and the computation power involved are considerably more than our suggested approach here.

We shall now see how this equivalent principle can be applied to optimize the reduced cloak. Consider the virtual isotropic inhomogeneous equivalent object, in order for it to have small scattering, it is highly desirable that the surface impedance matches that of the air. The equivalent object has impedance at the outer boundary of

$$
\eta(b)=g(b) .
$$

Clearly, by choosing $g(b)=1$, we will have a reduced cloak with little scattering.

We shall now show that the parameters of several published reduced cloaks (or in fact any cloak) can actually be derived from (3). We shall first present the linear mapping function, ${ }^{8}$

$$
f(r)= \begin{cases}\frac{r_{0}}{a} r, & 0<r \leq a, \\ b-\frac{b-r_{0}}{b-a}(b-r), & a<r \leq b, \\ r, & r>b .\end{cases}
$$

Substituting (7) into (3) and setting $g(r)=f(r) / r$ and $r_{0}=0$, we obtain the parameters of the reduced cloak proposed by Yan et al. ${ }^{12}$ Since this reduced cloak fulfils (6), one can anticipate that it induces little scattering. Substituting (7) into (3) and setting $g(r)=f(r) /\left[r f^{\prime}(r)\right]$ and $r_{0}=0$, we obtain the parameters of the reduced cloak first proposed by Cummer $e t$ $a l .{ }^{3}$ and eventually realized experimentally. ${ }^{5}$ It can be shown that the impedance of the equivalent isotropic inhomogeneous object for Cummer's cloak at the outer boundary is $(1-a / b)$, which is mismatched if the wave is coming from air, and as a result, it will cause some scattering and reflection. Instead, it is easy to see from the perspective of the isotropic inhomogeneous object that a better mapping function that satisfies (6) [i.e., $\left.f^{\prime}(b)=1\right]$ can be used to reduce the scattering and reflection. For TM wave, substituting (7) into (3) (with $\left\{\mu_{r} \rightarrow \varepsilon_{r}, \mu_{\theta} \rightarrow \varepsilon_{\theta}, \varepsilon_{z} \rightarrow \mu_{z}\right\}$ ) and setting $g(r)$ $=f(r) f^{\prime}(r) / r$, we obtain the parameters for the nonmagnetic reduced cloak ${ }^{7}$ proposed by Cai et al. Again, from our perspective, a better mapping function that satisfies (6) (i.e., $\left.f^{\prime}(b)=1\right)$ can be used to reduce the scattering and reflection. This is precisely their later version of the non-magnetic cloak, ${ }^{7}$ i.e., a nonlinear mapping which was chosen such that $f^{\prime}(b)=1$. They found that the scattering is reduced substantially due to the impedance matching at the outer boundary of the cloak. What they have done is that they defined the principal impedance $\eta_{r}=\sqrt{\mu_{z} / \varepsilon_{\theta}}=\sqrt{1 / \varepsilon_{\theta}}$, and set $\varepsilon_{\theta}=1$ at the outer boundary in order to match the impedance of the air background. In the same spirit, the impedance-matched hyperlens ${ }^{24}$ were also proposed. In general, there are two principal impedances, one is $\eta_{r}$ and the other one is $\eta_{\theta}$ $=\sqrt{\mu_{z} / \varepsilon_{r}}=\sqrt{1 / \varepsilon_{r}}$. By keeping both $\eta_{r}$ and $\eta_{\theta}$ to match the impedance of the air background, the impedance-matched reduced cloak is obtained. However, for a general form of the reduced cloak, one cannot define a unique impendence for the outer boundary because $\eta_{r} \neq \eta_{\theta}$ in general. For example, for the first version of nonmagnetic reduced cloak, $\eta_{r}(b)=(1-a / b)$ but $\eta_{\theta}(b)=1$. Using the present perspective, we can define the total impedance of this non-magnetic reduced cloak as that of the equivalent isotropic inhomogeneous object described above, which is $\eta(b)=b /(b-a)$ [but not $(1-a / b)$ as shown in Ref. 6]. In the later version of the nonmagnetic reduced cloak, one has $\eta(b)=1$ so that $\eta_{r}(b)$ $=\eta_{\theta}(b)=1$, consequently there is no ambiguity in defining a total impendence for the outer boundary. We shall emphasize that the impedance matching non-magnetic reduced cloak (or the impedance-matched hyperlens) are of considerable importance, and our current work here provides a deeper understanding in the transformation optics itself.

We will give one concrete numerical example to illustrate the equivalent between the reduced cloak and the isotropic inhomogeneous object. The commercial finite-element solver COMSOL MULTIPHYSICS was employed in our numerical calculation. A TE plane wave is incident from the left with a wavelength of $0.1 \mathrm{~m}$. We consider a reduced cloak with the parameters $r_{0}=0.05 m, a=0.1 m$, and $b=0.2 m$ in (7), and $g(r)=f(r) / r$. The resultant constitutive parameters are

$$
\mu_{r}=\frac{b-r_{0}}{b-a}\left(\frac{r-a^{\prime}}{r}\right)^{2}, \mu_{\theta}=\frac{b-r_{0}}{b-a}, \varepsilon_{z}=\frac{b-r_{0}}{b-a},
$$

for $a<r<b$ where $a^{\prime}=b\left(a-r_{0}\right) /\left(b-r_{0}\right)$. We also include an object for $r<a$ :

$$
\mu_{r}=\mu_{\theta}=\varepsilon_{z}=r_{0} / a,
$$

based on the same choice of $g(r)$. These parameters are very similar to the general parameters of the three dimensional ideal cloak. Consequently, we would like to call this case the "near ideal cloak."12 According to our analysis, the above reduced cloak together with the enclosed object is equivalent to an isotropic inhomogeneous object with constitutive parameters 

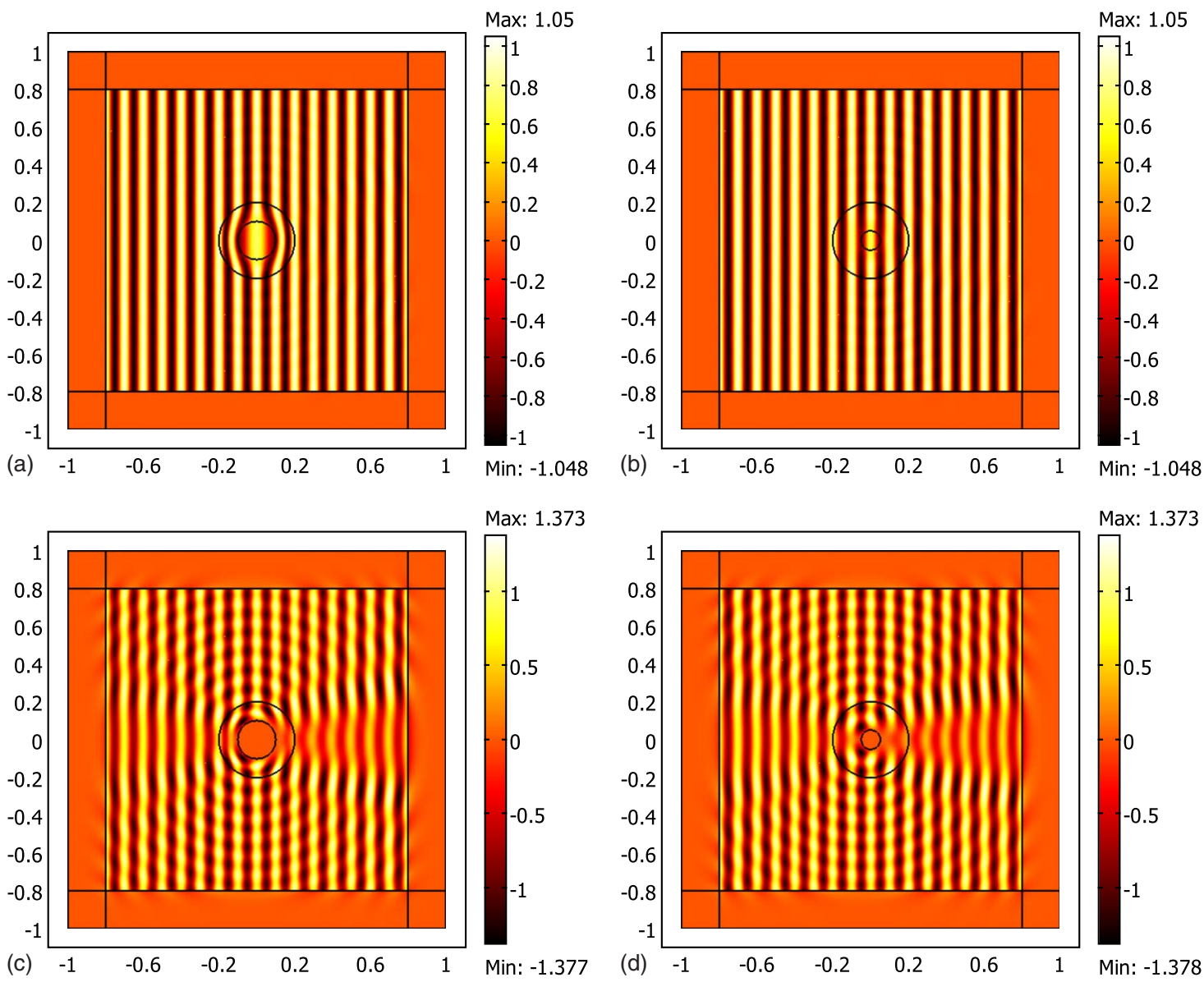

FIG. 1. (Color online) The electric field distribution of the reduced cloak and its equivalent isotropic inhomogeneous object. (a) the reduced cloak (8) and its cloaked object (9). (b) the equivalent isotropic inhomogeneous object corresponding to (a), with its parameters described by (10). (c) the reduced cloak described by (8), with a PEC inside it. (d) the equivalent isotropic inhomogeneous object corresponding to (c).

$$
\varepsilon=1 / \mu=\left\{\begin{array}{cc}
{\left[b-\frac{b-a}{b-r_{0}}(b-\rho)\right] / \rho,} & r_{0}<\rho<b, \\
a / r_{0}, & \rho<r_{0} .
\end{array}\right.
$$

Figure 1(a) shows the electric field distribution of the reduced cloak (8) and its enclosed object (9), which shows almost no scattering, as least to ours eyes. In Fig. 1(b), we plot the electric field distribution of the isotropic inhomogeneous object as described by (10). It is clear that for $r>b$, the scattering pattern is identical to that of Fig. 1(a). In either case, the vanishingly small scattering is induced by the matched impedance at all the interfaces. To have a deeper understanding, the cloaked object described in (9) and (10) is replaced by a perfect electric conductor (PEC) cylinder, and the scattering pattern is plotted in Figs. 1(c) and 1(d). In this case, the impedance mismatch at the inner boundary of the cloak induces strong scattering. We note that this strong scattering is a consequence of the relatively large $r_{0}$ that we have chosen. The reduced cloak can do a much better job if a smaller $r_{0}$ is used. In the case of $r_{0}=0$, we recover the reduced cloaks of Yan et al. ${ }^{12}$ It is quite clear that the identical far-field $(r>b)$ scattering patterns in Figs. 1(c) and 1(d) demonstrate the equivalence again.

Since calculating the scattering properties of an isotropic inhomogeneous object is considerably easier than that of the reduced cloak, our perspective provides an alternative and easier way to obtain the scattering properties of the reduced cloaks semi-analytically. In addition, within the framework of our understanding, we can also explain why different reduced cloaks work differently. We note that most studies for two dimensional reduced cloaks focused on the interactions between the reduced cloaks and normal incident waves (only three parameters are involved in such cases), and does not discuss the effect of oblique incidence. ${ }^{13,35,36}$ From a threedimensional perceptive, our approach also suggests a more straightforward way to design reduced cloaks and study the interactions between the reduced cloaks and the oblique incident waves by performing the coordinate transformation on Eqs. (4) and (5) and thereby the six parameters needed for the new set of the reduced cloaks after transformation are readily obtained. Whether the "reduction process" still gives good cloaking properties for oblique incidence would then be more transparent, but of course the conclusion will depend on the form of $g(r)$ and $f(r)$ being chosen. Some will be better than others. 
In conclusion, we found that the general two-dimensional reduced cloak is directly linked to an isotropic inhomogeneous object by exactly the coordinate transformation that one applies to the ideal cloak. This perspective serves as a bridge between some of the formerly introduced reduced cloaks and the original transformation optics. Moreover, it also provides an alternative way to calculate the scattering properties of the reduced cloaks.

This work was supported by Hong Kong RGC Grant No. 600209. Computation resources are supported by Shun Hing Education and Charity Fund. *kenyon@ust.hk

†phchan@ust.hk

${ }^{1}$ U. Leonhardt, Science 312, 1777 (2006).

${ }^{2}$ J. B. Pendry, D. Schurig, and D. R. Smith, Science 312, 1780 (2006).

${ }^{3}$ S. A. Cummer, B.-I. Popa, D. Schurig, D. R. Smith, and J. B. Pendry, Phys. Rev. E 74, 036621 (2006).

${ }^{4}$ D. Schurig, J. B. Pendry, and D. R. Smith, Opt. Express 14, 9794 (2006).

${ }^{5}$ D. Schurig, J. J. Mock, B. J. Justice, S. A. Cummer, J. B. Pendry, A. F. Starr, and D. R. Smith, Science 314, 977 (2006).

${ }^{6}$ W. Cai, U. K. Chettiar, A. V. Kildishev, and V. M. Shalaev, Nat. Photonics 1, 224 (2007).

${ }^{7}$ W. Cai, U. K. Chettiar, A. V. Kildishev, V. M. Shalaev, and G. W. Milton, Appl. Phys. Lett. 91, 111105 (2007).

${ }^{8}$ H. Y. Chen, Z. Liang, P. Yao, X. Jiang, H. Ma and C. T. Chan, Phys. Rev. B 76, 241104(R) (2007).

${ }^{9}$ H. S. Chen, B.-I. Wu, B. Zhang, and J. A. Kong, Phys. Rev. Lett. 99, 063903 (2007).

${ }^{10}$ Z. Ruan, M. Yan, C. W. Neff, and M. Qiu, Phys. Rev. Lett. 99, 113903 (2007).

${ }^{11}$ M. Yan, Z. Ruan, and M. Qiu, Phys. Rev. Lett. 99, 233901 (2007).

${ }^{12}$ M. Yan, Z. Ruan, and M. Qiu, Opt. Express 15, 17772 (2007).

${ }^{13}$ B. Zhang, H. S. Chen, and B.-I. Wu, Opt. Express 16, 14655 (2008).

${ }^{14}$ J. Li and J. B. Pendry, Phys. Rev. Lett. 101, 203901 (2008).

${ }^{15}$ U. Leonhardt and T. Tyc, Science 323, 110 (2009).

${ }^{16}$ R. Liu, C. Ji, J. J. Mock, J. Y. Chin, T. J. Cui, and D. R. Smith, Science 323, 366 (2009).

${ }^{17}$ J. Valentine, J. Li, T. Zentgraf, G. Bartal, and X. Zhang, Nature Mater. 8, 568 (2009).
${ }^{18}$ L. H. Gabrielli, J. Cardenas, C. B. Poitras, and M. Lipson, Nat. Photonics 3, 461 (2009).

${ }^{19}$ M. Rahm, D. Schurig, D. A. Roberts, S. A. Cummer, D. R. Smith, and J. B. Pendry, Photonics Nanostruct. Fundam. Appl. 6, 87 (2008).

${ }^{20}$ H. Y. Chen and C. T. Chan, Appl. Phys. Lett. 90, 241105 (2007).

${ }^{21}$ Y. Luo, H. S. Chen, J. Zhang, L. Ran, and J. A. Kong, Phys. Rev. B 77, 125127 (2008).

${ }^{22}$ H. Y. Chen, B. Hou, S. Chen, X. Ao, W. Wen, and C. T. Chan, Phys. Rev. Lett. 102, 183903 (2009).

${ }^{23}$ A. Greenleaf, Y. Kurylev, M. Lassas, and G. Uhlmann, Phys. Rev. Lett. 99, 183901 (2007).

${ }^{24}$ A. V. Kildishev and E. E. Narimanov, Opt. Lett. 32, 3432 (2007).

${ }^{25}$ M. Rahm, S. A. Cummer, D. Schurig, J. B. Pendry, and D. R. Smith, Phys. Rev. Lett. 100, 063903 (2008).

${ }^{26}$ H. Y. Chen and C. T. Chan, Phys. Rev. B 78, 054204 (2008).

${ }^{27}$ H. Y. Chen, X. Luo, H. Ma, and C. T. Chan, Opt. Express 16, 14603 (2008).

${ }^{28}$ M. Yan, W. Yan, and M. Qiu, Phys. Rev. B 78, 125113 (2008).

${ }^{29}$ T. Yang, H. Y. Chen, X. Luo, and H. Ma, Opt. Express 16, 18545 (2008).

${ }^{30}$ J. Ng, H. Y. Chen, and C. T. Chan, Opt. Lett. 34, 644 (2009).

${ }^{31}$ Y. Lai, H. Y. Chen, Z. Q. Zhang, and C. T. Chan, Phys. Rev. Lett. 102, 093901 (2009).

${ }^{32}$ A. Cho, Science 323, 701 (2009).

${ }^{33}$ H. Y. Chen, J. Opt. A, Pure Appl. Opt. 11, 075102 (2009).

${ }^{34}$ P. C. Waterman, Phys. Rev. D 3, 825 (1971).

${ }^{35}$ B. Zhang, H. Chen, B.-I. Wu, Y. Luo, L. Ran, and J. A. Kong, Phys. Rev. B 76, 121101(R) (2007).

${ }^{36}$ S. Xi, H. S. Chen, B. Zhang, B.-I. Wu, and J. A. Kong, Phys. Rev. B 79, 155122 (2009). 